\title{
Continuum model of magnetic field induced viscoelasticity in magnetorheological fluids
}

Cite as: J. Chem. Phys. 150, 174901 (2019); https://doi.org/10.1063/1.5090337

Submitted: 26 January 2019 . Accepted: 12 April 2019 . Published Online: 06 May 2019

Tilen Potisk (D), Daniel Svenšek $(D$, Harald Pleiner, and Helmut R. Brand
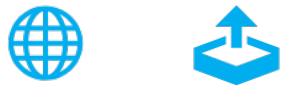

\section{ARTICLES YOU MAY BE INTERESTED IN}

\section{Electronic friction in interacting systems}

The Journal of Chemical Physics 150, 174101 (2019); https://doi.org/10.1063/1.5095425

Calibration of the Flory-Huggins interaction parameter in field-theoretic simulations The Journal of Chemical Physics 150, 174902 (2019); https://doi.org/10.1063/1.5089217

Electron transfer in confined electromagnetic fields

The Journal of Chemical Physics 150, 174122 (2019); https://doi.org/10.1063/1.5095940

The Journal 


\title{
Continuum model of magnetic field induced viscoelasticity in magnetorheological fluids
}

\author{
Cite as: J. Chem. Phys. 150, 174901 (2019); doi: 10.1063/1.5090337 \\ Submitted: 26 January 2019 • Accepted: 12 April 2019 • \\ Published Online: 3 May 2019
}

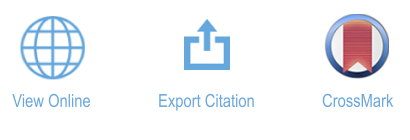

Tilen Potisk, ${ }^{1, a)}$ (Daniel Svenšek, ${ }^{2}$ (D) Harald Pleiner, ${ }^{3}$ and Helmut R. Brand ${ }^{7}$

\author{
AFFILIATIONS \\ ${ }^{1}$ Department of Physics, University of Bayreuth, 95440 Bayreuth, Germany \\ ${ }^{2}$ Department of Physics, Faculty of Mathematics and Physics, University of Ljubljana, SI-1000 Ljubljana, Slovenia \\ ${ }^{3}$ Max Planck Institute for Polymer Research, 55021 Mainz, Germany
}

a) Electronic mail: tilen.potisk@uni-bayreuth.de

\begin{abstract}
An effective macroscopic model of magnetorheological fluids in the viscoelastic regime is proposed. Under the application of an external magnetic field, columns of magnetizable particles are formed in these systems. The columns are responsible for solidlike properties, such as the existence of elastic shear modulus and yield stress, and are captured by the strain field, while magnetic properties are described by the magnetization. We investigate the interplay of these variables when static shear or normal pressure is imposed in the presence of the external magnetic field. By assuming a relaxing strain field, we calculate the flow curves, i.e., the shear stress as a function of the imposed shear rate, for different values of the applied magnetic field. Focusing on the small amplitude oscillatory shear, we study the complex shear modulus, i.e., the storage and the loss moduli, as a function of the frequency. We demonstrate that already such a minimal model is capable of furnishing many of the key physical features of these systems, such as yield stress, enhancement of the shear yield stress by pressure, threshold behavior in the spirit of the frequently employed Bingham law, and several features in the frequency dependence of storage and loss moduli.
\end{abstract}

Published under license by AIP Publishing. https://doi.org/10.1063/1.5090337

\section{INTRODUCTION}

Magnetorheological (MR) fluids are a class of fluids, which experience significant changes upon application of an external magnetic field. Examples of such changes are a fast and reversible acquisition of solidlike properties and a dramatic increase in the viscosity. This makes such systems suitable for many applications such as shock absorbers, clutches, and brakes.

Introduced in Ref. 1, MR fluids are composed of micron sized magnetizable particles, typically suspended in oil. Under the influence of the external magnetic field, gap-spanning chains or columns of the particles in the direction of the field are formed and a finite stress, also called the yield stress, is needed to break these structures and initiate flow. The columnar structure can be explained by the induced dipole interaction between the particles, which is attractive when they are parallel to the magnetic field. The application of the magnetic field also leads to a significant rise in the viscosity. For reviews of general aspects of MR fluids, cf. Refs. 2-6.
Several microscopic and macroscopic models have been developed to predict the column formation and the dependence of the yield stress on the applied magnetic field. Due to the similar form of the interaction between the particles (dipolar), the studies of the electrorheological fluids are also relevant for the description of MR fluids. Microscopic models usually assumed single chain structures, which are deformed in the shear plane, ${ }^{7-11}$ although certain refinements on the electrorheological fluids have been done taking into account the crystal structure of the particle aggregates. ${ }^{12}$ Theoretical studies of rheological properties are much less frequent. In Ref. 13, the so-called independent droplet model was used to model the shear-thinning behavior. Other studies focus on single chains ${ }^{14,15}$ or simulations of individual particles. ${ }^{1}$

Macroscopic models usually rely on the Maxwell stress tensor and magnetostriction effects. In the case of electrorheological fluids, the anisotropy caused by the chains was treated using a directorlike degree of freedom known in nematic liquid crystals. ${ }^{17-19}$ In Ref. 20, the static yield stress was calculated for a fluid with lamellar 
structure. To predict the rheological properties, the two fluid approach, where the solvent phase and the particle phase are treated separately, has been used. ${ }^{21,22}$ With this approach, various aspects of pattern formation including sheets, disktype structures in a rotating field, etc., have also been analyzed. ${ }^{2}$

The aim of this paper is to construct a minimal macroscopic model for MR fluids, which is capable of capturing the main physical effects found in static as well as dynamic experiments. To derive the static and the dynamic equations, a symmetry based approach is used. ${ }^{26}$ One of its advantages with respect to microscopic approaches is the applicability to different systems and geometries.

This article is organized as follows: The macroscopic model is introduced in Sec. II, followed by the numerical analysis of the static shear deformation in the external magnetic field in Sec. III A. In Sec. III B, the effects of the normal pressure on the static yield stress are considered. Flow properties are discussed by analyzing shear stresses due to stationary (Sec. IV A) and oscillatory (Sec. IV B) imposed shear flow.

\section{MACROSCOPIC MODEL}

Generally, in a fluid mixture, the macroscopic variables are mass density $\rho$, momentum density g, entropy density $\sigma$, and concentration density $c$. For magnetic fluids, there is, in addition, a magnetization field $\mathbf{M}$, which is zero in equilibrium in the absence of an external magnetic field. For MR fluids, in particular, an external field triggers columnar structures of the magnetizable particles due to the attractive magnetic forces between the particles that lead to solidlike, elastic properties. Therefore, we introduce a strain field $\varepsilon_{i j}$ as a macroscopic variable that is zero in the field-free case and finite in a magnetic field. For the dynamics, we restrict ourselves to the regime, where the viscoelastic nature of the MR fluids can be described by a relaxational dynamics for the strain field.

In order to make the model as simple as possible, we disregard the density $\rho$, the entropy density $\sigma$, and the concentration $c$ as variables, effectively meaning they are constant. This implies incompressibility and the neglect of temperature and concentration gradients (sedimentation). Thus, we deal with elastic deformations and flow, as well as the magnetization. Furthermore, we will consider all material tensors only in their isotropic form. This seems to be a reasonable simplification, since we only consider shear in the plane perpendicular to the magnetization (and compression along the field). It turns out that the static and the dynamic theoretical behavior is in qualitative agreement with experiments. Even the anisotropy of the ultrasound velocity can be explained without invoking anisotropic material tensors. ${ }^{27-29}$ We emphasize that the introduction of a director in the present context is inappropriate, since it has the wrong behavior under time reversal. If anisotropy is taken into account, all the material tensors acquire additional terms due to the lower symmetry. Furthermore, in an elastic system with an orientational order, one must, in principle, consider the additional variable of relative rotations between the network and the preferred direction. These relative rotations play an important role in the description of nematic gels. ${ }^{30,31}$ Throughout most of this paper, we concentrate on the isotropic aspects of material properties and, therefore, discard relative rotations.

\section{A. Statics}

The statics of a macroscopic system is best set up by considering its total energy density $\epsilon$. The Gibbs relation, a manifestation of the first law of thermodynamics, relates changes of the macroscopic variables to energy changes

$$
\mathrm{d} \epsilon=\mathrm{d} \epsilon_{0}+v_{i} \mathrm{~d} g_{i}+h_{i}^{M} \mathrm{~d} M_{i}+\psi_{i j} \mathrm{~d} \varepsilon_{i j},
$$

where $\mathrm{d} \epsilon_{0}$ represents the neglected macroscopic degrees of freedom and is given in Ref. 26. The thermodynamic conjugates to the macroscopic variables considered here are the bulk velocity $v_{i}$, the magnetic molecular field $h_{i}^{M}$, and the elastic stress $\psi_{i j}$.

The statics is described by the energy density $\epsilon\left(\varepsilon_{i j}, \mathbf{M}, \mathbf{g}\right)$,

$$
\begin{aligned}
\epsilon= & \epsilon_{0}-\mu_{0} H_{i} M_{i}+\frac{1}{2} \alpha \mathbf{M}^{2}+\frac{1}{4} \beta\left(\mathbf{M}^{2}\right)^{2} \\
& +\frac{1}{2} c_{i j k l} \varepsilon_{i j} \varepsilon_{k l}-\frac{1}{2} \gamma_{i j k l} \varepsilon_{i j} M_{k} M_{l}+\frac{1}{2 \rho} \mathbf{g}^{2},
\end{aligned}
$$

where the coupling to an external magnetic field, $\sim \mu_{0} \mathbf{H}$, ensures the induced magnetization to be parallel to the field, while the next two terms govern the magnitude of the magnetization (modulus) $M \equiv \sqrt{\mathbf{M}^{2}}$ that is induced by the field. The form given in Eq. (2) is suitable for rather small fields, while in the general case, the $\alpha$ and $\beta$ terms have to be replaced by a more complicated function $f_{1}\left(M^{2}\right)$ that can be taken from experimental results.

The material tensors $c_{i j k l}$ and $\gamma_{i j k l}$ describe elasticity and magnetostriction, respectively. In their standard isotropic form, ${ }^{32}$

$$
\begin{aligned}
& c_{i j k l}=c_{1} \mathbf{M}^{2} \delta_{i j} \delta_{k l}+c_{2} \mathbf{M}^{2}\left(\delta_{i k} \delta_{j l}+\delta_{i l} \delta_{k j}\right), \\
& \gamma_{i j k l}=\gamma_{1} \delta_{i j} \delta_{k l}+\gamma_{2}\left(\delta_{i k} \delta_{j l}+\delta_{i l} \delta_{k j}\right),
\end{aligned}
$$

where we have assumed here that the elastic moduli are proportional to $M^{2}$. This ensures that elasticity, and therefore the elastic tensor $c_{i j k l}$, vanishes, when there are no columns, i.e., when the magnetization $\mathbf{M}$ is zero. This quadratic dependence is the simplest assumption but can be replaced by a more complicated (even discontinuous) function $f_{2}\left(M^{2}\right)$, provided $f_{2}\left(M^{2}\right) \rightarrow 0$ for $M \rightarrow 0$. The dependence on $M^{2}$ (rather than $M_{i}$ ) is due to the time reversal behavior of $\mathbf{M}$.

As usual, the isotropic tensors $c_{i j k l}$ and $\gamma_{i j k l}$ have two coefficients each, where the terms $\sim c_{1}$ and $\sim \gamma_{1}$ describe the energy associated with the compressive or elongational strains, while the coefficients $c_{2}$ and $\gamma_{2}$ correspond to the shear strains. In the examples described in Secs. III A and III B we assume that the compression is always parallel to the magnetic field and that for the shear deformation the shear plane contains the magnetic field. For completeness, if uniaxial anisotropy of the system is considered, with the axis along $\mathbf{m}=\mathbf{M} /|\mathbf{M}|$, one gets additional terms in Eqs. (3) and (4) $)^{33}$

$$
\begin{aligned}
\tilde{c}_{i j k l}= & \tilde{c}_{1} \delta_{i j}^{\perp} \delta_{k l}^{\perp}+\tilde{c}_{2}\left(\delta_{i k}^{\perp} \delta_{j l}^{\perp}+\delta_{i l}^{\perp} \delta_{k j}^{\perp}-\delta_{i j}^{\perp} \delta_{k l}^{\perp}\right)+\tilde{c}_{3} m_{i} m_{j} m_{k} m_{l} \\
& +\tilde{c}_{4}\left(m_{i} m_{j} \delta_{k l}^{\perp}+m_{k} m_{l} \delta_{i j}^{\perp}\right)+\tilde{c}_{5}\left(m_{i} m_{k} \delta_{j l}^{\perp}+m_{i} m_{l} \delta_{j k}^{\perp}\right. \\
& \left.+m_{j} m_{k} \delta_{i l}^{\perp}+m_{j} m_{l} \delta_{i k}^{\perp}\right), \\
\tilde{\gamma}_{i j k l}= & \tilde{\gamma}_{1} \delta_{i j}^{\perp} \delta_{k l}^{\perp}+\tilde{\gamma}_{2}\left(\delta_{i k}^{\perp} \delta_{j l}^{\perp}+\delta_{i l}^{\perp} \delta_{k j}^{\perp}-\delta_{i j}^{\perp} \delta_{k l}^{\perp}\right)+\tilde{\gamma}_{3} m_{i} m_{j} m_{k} m_{l} \\
& +\tilde{\gamma}_{4} m_{i} m_{j} \delta_{k l}^{\perp}+\tilde{\gamma}_{5} m_{k} m_{l} \delta_{i j}^{\perp}+\tilde{\gamma}_{6}\left(m_{i} m_{k} \delta_{j l}^{\perp}+m_{i} m_{l} \delta_{j k}^{\perp}\right. \\
& \left.+m_{j} m_{k} \delta_{i l}^{\perp}+m_{j} m_{l} \delta_{i k}^{\perp}\right),
\end{aligned}
$$


where $\delta_{i j}^{\perp}=\delta_{i j}-m_{i} m_{j}$ and where $\tilde{c}_{1}, \tilde{c}_{2}, \tilde{c}_{3}, \tilde{c}_{4}$, and $\tilde{c}_{5}$ are all proportional to $\mathbf{M}^{2}$. As already mentioned above, here we will not pursue these refinements and will be using the isotropic forms [Eqs. (3) and (4)].

To calculate the equilibrium values of the variables, one must first calculate the thermodynamic forces. These are the thermodynamic conjugates of the macroscopic variables [Eq. (1)] and are derived by taking the variational derivatives of the energy density [Eq. (2)] with respect to the corresponding variables ${ }^{26}$

$$
\begin{gathered}
h_{i}^{M}=\frac{\delta \epsilon}{\delta M_{i}}=\left(\alpha+\beta \mathbf{M}^{2}\right) M_{i}-\mu_{0} H_{i}-\gamma_{1} M_{i} \varepsilon_{k k}-2 \gamma_{2} M_{j} \varepsilon_{i j} \\
+\left(c_{1} \varepsilon_{k k}^{2}+2 c_{2} \varepsilon_{k j} \varepsilon_{j k}\right) M_{i}, \\
\psi_{i j}=\frac{\delta \epsilon}{\delta \varepsilon_{i j}}=c_{1} \varepsilon_{k k} \mathbf{M}^{2} \delta_{i j}+2 c_{2} \mathbf{M}^{2} \varepsilon_{i j}-\frac{1}{2} \gamma_{1} \mathbf{M}^{2} \delta_{i j}-\gamma_{2} M_{i} M_{j}, \\
v_{i}=\frac{\delta \epsilon}{\delta g_{i}}=\frac{1}{\rho} g_{i} .
\end{gathered}
$$

Thermodynamic equilibrium requires all thermodynamic forces to be zero, i.e., $h_{i}^{M}=0$ and $\psi_{i j}=0$. For a finite external magnetic field, $H_{i}=H \delta_{i z}$, the conditions (7)-(9) lead to a finite equilibrium magnetization $M_{z}^{e q}=\mu_{0} H / \alpha$ and a finite equilibrium strain $\varepsilon_{z z}^{e q}=(1 / 2)\left(\gamma_{1}+2 \gamma_{2}\right) /\left(c_{1}+2 c_{2}\right)$. The latter is independent of the field since the field dependences of the magnetostriction and the elasticity compensate each other. Note that our simplified linear model only applies for finite fields and does not describe the case $H \equiv 0$.

\section{B. Macroscopic dynamics}

The dynamic evolution of deviations from the equilibrium state is described by the proper macroscopic equations discussed in the following. The dynamic equations for the momentum density $g_{i}$, the magnetization $M_{i}$, and the strain field $\varepsilon_{i j}$ are ${ }^{32}$

$$
\begin{gathered}
\frac{d}{d t} g_{i}+\nabla_{j}\left(p \delta_{i j}-\psi_{i j}+\sigma_{i j}^{t h}+\sigma_{i j}\right)=0, \\
\frac{d}{d t} M_{i}+\epsilon_{i j k} M_{j} \omega_{k}+X_{i}=0, \\
\frac{d}{d t} \varepsilon_{i j}+\varepsilon_{k j} \nabla_{i} v_{k}+\varepsilon_{k i} \nabla_{j} v_{k}-A_{i j}+Y_{i j}=0,
\end{gathered}
$$

where $d /(d t) \equiv \partial /(\partial t)+v_{j} \nabla_{j}$ is the material derivative, $A_{i j}=\left(\nabla_{i} v_{j}+\nabla_{j} v_{i}\right) / 2$ is the symmetric gradient of the velocity field, and the vorticity $\omega_{i}=\epsilon_{i j k} \nabla_{j} v_{k} / 2$ corresponds to its antisymmetric gradient.

The thermodynamic pressure, $p$, is given $b^{26}$

$$
p=-\epsilon+v_{i} g_{i}+B_{i} H_{i}+\epsilon_{0},
$$

where $\mathbf{B}=\mu_{0}(\mathbf{H}+\mathbf{M})$ is the magnetic flux density and $\epsilon_{0}$ represents the neglected macroscopic degrees of freedom. The nonlinear stress tensor contributions read in symmetrized form ${ }^{32}$

$$
\sigma_{i j}^{t h}=-\frac{1}{2}\left(B_{i} H_{j}+B_{j} H_{i}\right)+\frac{1}{2}\left(\psi_{j k} \varepsilon_{k i}+\psi_{i k} \varepsilon_{k j}\right) .
$$

The nonphenomenological parts of the currents, shown explicitly in Eqs. (10)-(14), are not related to any phenomenological (transport) parameters and are given by general symmetry and thermodynamic principles. ${ }^{26}$ We emphasize that their structure, in particular, that of the convective derivative $\varepsilon_{k j} \nabla_{i} v_{k}+\varepsilon_{k i} \nabla_{j} v_{k}$ in Eq. (12), is uniquely determined. ${ }^{34,35}$ All those terms are reversible, meaning that they transform under time reversal, $t \rightarrow-t$, in the same way as the time derivative of their appropriate variable. For a general discussion of time reversal symmetry and its importance for macroscopic equations, we refer to Ref. 36 .

The phenomenological part of the stress tensor $\sigma_{i j}$ and the quasicurrents $X_{i}$ and $Y_{i j}$ describe temporal changes of their corresponding variables and can be written as a sum of a reversible (superscript R) and an irreversible part (superscript D). They are functions of the thermodynamic forces [Eqs. (7)-(9)] $\left(A_{i j}, h_{i}^{M}\right.$, and $\psi_{i j}$ ) involving phenomenological transport parameters. The second law of thermodynamics states that irreversible dynamic processes always dissipate energy (transfer energy to the microscopic degrees of freedom as heat) and therefore increase the entropy. On the contrary, reversible processes are nondissipative and must not increase the entropy.

Within linear irreversible thermodynamics, ${ }^{37}$ the dissipation function $R$, which is proportional to the entropy production, can be written as a bilinear form of fluxes and forces, in our case

$$
2 R=-\sigma_{i j} A_{i j}+X_{i} h_{i}^{M}+Y_{i j} \psi_{j i}
$$

For the reversible parts of the currents $\left\{\sigma_{i j}^{R}, X_{i}^{R}, Y_{i j}^{R}\right\}$, one has to require $R=0$, while the dissipative ones $\left\{\sigma_{i j}^{D}, X_{i}^{D}, Y_{i j}^{D}\right\}$ fulfill $R>0$.

To derive the dissipative parts of the (quasi-) currents, one writes the dissipation function $R$ as a quadratic form in the relevant thermodynamic forces. By taking the variational derivative of this function with respect to the chosen thermodynamic force, according to Eq. (15), one gets the corresponding dissipative current. The dissipation function is

$$
R=\frac{1}{2} v_{i j k l}^{D} A_{i j} A_{k l}+\frac{1}{2} b^{D} h_{i}^{M} h_{i}^{M}+\frac{1}{2}(1 / \tau)_{i j k l} \psi_{i j} \psi_{k l}+d_{i j k} \psi_{j k} h_{i}^{M},
$$

leading to

$$
\begin{aligned}
\sigma_{i j}^{D} & =-v_{i j k l}^{D} A_{k l}, \\
X_{i}^{D} & =b^{D} h_{i}^{M}+d_{i j k} \psi_{j k}, \\
Y_{i j}^{D} & =(1 / \tau)_{i j k l} \psi_{k l}+d_{k i j} h_{k}^{M} .
\end{aligned}
$$

As in Eqs. (3) and (4), we again assume an isotropic form of the material tensors $v_{i j k l}^{D}$ and $(1 / \tau)_{i j k l}$ describing viscosity and strain relaxation, respectively,

$$
\begin{gathered}
v_{i j k l}^{D}=v_{1} \delta_{i j} \delta_{k l}+v_{2}\left(\delta_{i k} \delta_{j l}+\delta_{j k} \delta_{i l}\right), \\
(1 / \tau)_{i j k l}=\frac{1}{\tau_{1} M_{0}^{2}} \delta_{i j} \delta_{k l}+\frac{1}{\tau_{2} M_{0}^{2}}\left(\delta_{i k} \delta_{j l}+\delta_{j k} \delta_{i l}\right) .
\end{gathered}
$$

Thus, we model the viscoelastic properties of MR fluids ${ }^{38-43}$ by using the strain field as a relaxing variable. When the columns are deformed, the particles experience a drive to redistribute, e.g., by permeation effects, which shows on the macroscopic level 
(a)

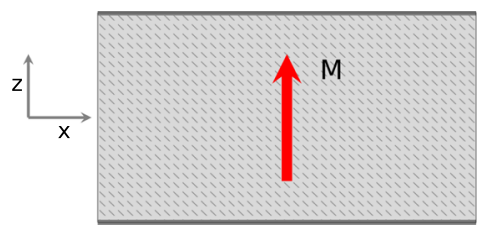

(b)

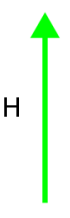

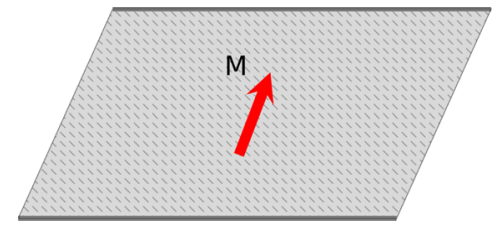

FIG. 1. Sketch of a MR fluid, confined between two parallel plates. (a) The external magnetic field is applied perpendicularly to the plates, which induces a nonzero magnetization (red arrow) along the $z$ axis. (b) MR fluid, when an additional static shear deformation is imposed.

as relaxation of the strain. In addition, we assume that the relaxation coefficients, $\tau_{1} M_{0}^{2}$ and $\tau_{2} M_{0}^{2}$, are proportional to $M_{0}^{2}$, which is motivated by the fact that the elastic network relaxes on longer time scales in larger magnetic fields, i.e., it behaves more elastically. Depending on the type of deformation, either compression or shear, the strain relaxes with a characteristic time proportional to $\tau_{1}+2 \tau_{2}$ or $\tau_{2}$, respectively. It should be noted that $\tau_{1,2}$ can still be functions of $M^{2}$, which is also the case for the viscosities $v_{1,2}$ and the magnetization relaxation $b^{D}$. In the incompressible case, $v_{1}$ drops out and can be put to zero. We discard diffusiontype contributions $\sim \nabla_{k} \psi_{i j}$.

In Eq. (16), the material tensor

$$
d_{i j k}=d_{1} M_{i} \delta_{j k}+d_{2}\left(M_{j} \delta_{i k}+M_{k} \delta_{i j}\right)
$$

represents the dissipative coupling of the elastic stress to the magnetization. It is linear in the magnetic field to make sure that the contributions to $X_{i}^{D}$ and $Y_{i j}^{D}$ are irreversible, but an additional $M^{2}$ dependence is possible.

The reversible currents cannot be derived from the dissipation function since $R \equiv 0$ for the reversible case. Instead, they are set up by collecting all possible combinations allowed by (e.g., time-reversal) symmetry that leads to a vanishing $R$ in Eq. (15),

$$
\begin{aligned}
\sigma_{i j}^{R} & =-v_{i j k l}^{R} A_{k l}-c_{k i j}^{R} h_{k}^{M}, \\
X_{i}^{R} & =b_{i j}^{R} h_{j}^{M}-c_{i j k}^{R} A_{j k}, \\
Y_{i j}^{R} & =0 .
\end{aligned}
$$

The material tensors in Eqs. (23) and (24) $\operatorname{read}^{32}$

$$
\begin{aligned}
v_{i j k l}^{R} & =v^{R}\left(\epsilon_{i k p} \delta_{j l}+\epsilon_{j k p} \delta_{i l}+\epsilon_{i l p} \delta_{j k}+\epsilon_{j l p} \delta_{i k}\right) M_{p}, \\
c_{i j k}^{R} & =c_{1}^{R} M_{i} \delta_{j k}+c_{2}^{R}\left(M_{j} \delta_{i k}+M_{k} \delta_{i j}\right), \\
b_{i j}^{R} & =b^{R} \epsilon_{i j k} M_{k},
\end{aligned}
$$

where $c_{i j k}^{R}$ has the same form as $d_{i j k}$ in Eq. (22). To make these currents reversible, all three material tensors have to be odd functions in $M_{i}$ and additional $M^{2}$ dependences of the parameters are possible. In the incompressible case, $v^{R}$ and $c_{1}^{R}$ drop out and can be put to zero.

\section{Geometry and material parameters}

Throughout this paper, we use the geometry of two parallel plates, as shown in Fig. 1. The macroscopic variables are assumed to be only a function of the $z$ coordinate. The magnetic field will always be applied normal to the plates (along the $z$ axis). In Secs. III A and IV, we consider shear deformations as shown in Fig. 1(b), while in Sec. III B, a compressive strain (along the field direction) is additionally assumed.

Here, we list the values of the material parameters that we generally use in our numerical calculations (exceptions are indicated in the figure captions). The prefactors of the elastic moduli are $c_{1}=c_{2}$ $=10 \mathrm{~Pa} \mathrm{~A} \mathrm{~A}^{-2} \mathrm{~m}^{2}$, and the coefficients connected with the modulus of the magnetization are $\alpha=0.06 \mathrm{~Pa} \mathrm{~A}^{-2} \mathrm{~m}^{2}$ and $\beta=10^{-8} \mathrm{~Pa} \mathrm{~A}^{-4} \mathrm{~m}^{4}$. The values for $c_{2}$ and $\alpha$ are estimated by comparing the results of Sec. III [Eqs. (34) and (36)] to the measurements of the static (elastic) yield stress and the value of the critical strain in Ref. 44. For the magnetostrictive parameters, we find, by comparison of Eq. (38) with experimental results, $\gamma_{2} \approx 0.3 \mathrm{~Pa} \mathrm{~A}^{-2} \mathrm{~m}^{2}$, cf. Sec. III A, and $\gamma_{1}$ $\approx 1.2 \mathrm{~Pa} \mathrm{~A}^{-2} \mathrm{~m}^{2}$, see Eq. (42) in Sec. III B.

Among the transport parameters that we use are $v_{2}=0.2 \mathrm{~Pa}$ $\mathrm{s}, b^{D}=40 \mathrm{~A}^{2} \mathrm{~Pa}^{-1} \mathrm{~s}^{-1} \mathrm{~m}^{-2}$, and $\tau_{2}=0.1 \mathrm{~Pa} \mathrm{~s} \mathrm{~m} \mathrm{~A}^{-2}$ or $\tau_{2}=10 \mathrm{~Pa} \mathrm{~s} \mathrm{~m} \mathrm{~A}^{-2}$ in Sec. IV B. The coefficient, $\tau_{2}$, corresponding to the strain relaxation, is estimated from viscoelastic measurements, where, under a step shear strain deformation, the shear stress relaxed on the order of $0.01 \mathrm{~s}^{45}$ or $1 \mathrm{~s}^{38}$ This time is then compared to the characteristic time scale that shows up in our model, $\tau_{\mathrm{el}}=$ $\tau_{2} / 4 c_{2}$, cf. Sec. IV B. The reversible coupling coefficient, $c_{2}^{R}$, relating the magnetization to the symmetric velocity gradient is already known from the dynamics of magnetic liquids, ${ }^{46-48}$ where it was crucial to explain dynamic experiments. ${ }^{49,50}$ We use a similar value as in Ref. $50, c_{2}^{R}=0.4$.

\section{STATIC DEFORMATIONS}

We discuss first the relation between static deformations and elastic stresses for different magnetic field strengths. Since the strains are relaxing, purely static experiments can only be performed on time scales short compared to the strain relaxation time, where strains can effectively be described by the static equations of Sec. II A.

\section{A. Static shear deformation}

In this section, we study the static shear deformation, when the MR fluid is confined between two parallel plates and the upper plate is displaced parallel to the $x$ axis. Such a geometry is typically used in experiments to measure the elastic shear stress as a function of the shear strain. Experimental results show that the elastic shear stress first increases linearly with the shear strain, but when 
the shear strain is increased further, one typically observes a saturation in the elastic shear stress. The value of the elastic stress, where the stress-strain curve levels off, is known as static yield stress, and it is from the application point of view desirable to have it as large as possible. The value of the static yield stress was measured for many different MR fluids and it increases quadratically with the field for small fields. ${ }^{44}$ For intermediate magnetic fields, the static yield stress increases with the power of $3 / 2$, which was measured experimentally, ${ }^{43}$ as well as modeled numerically in Ref. 7 , taking into account the saturation effects of the magnetization of the particles. The static yield strain does not depend on the magnetic field and is typically around $0.5 \%$. $^{42,44}$

Throughout this section, we assume that the elastic shear deformation of Fig. 1(b) is constant, $\varepsilon_{x z}=\varepsilon_{z x}=\frac{1}{2} \Gamma$, and all other components are vanishing. This can be achieved by a displacement of the upper plate by $\mathbf{u}=\Gamma \hat{\mathbf{e}}_{x}$, where $\Gamma$ is called the shear strain.

The elastic shear stress induced by the shear deformation follows from Eq. (8), which now reads

$$
\psi_{x z}=c_{2} \mathbf{M}^{2} \Gamma-\gamma_{2} M_{x} M_{z},
$$

while the magnetization follows from Eq. (7)

$$
\begin{aligned}
\mu_{0} H & =\alpha M_{z}+c_{2} \Gamma^{2} M_{z}-\gamma_{2} \Gamma M_{x}, \\
0 & =\alpha M_{x}+c_{2} \Gamma^{2} M_{x}-\gamma_{2} \Gamma M_{z},
\end{aligned}
$$

where $\beta$ is neglected here.

These equations can be solved analytically, but the resulting formulas are rather involved. We will discuss and explain the main features either using special cases or show figures of numerical solutions.

In Fig. 2, we present the elastic shear stress as a function of the shear strain at three different values of the applied magnetic field. One can see that the elastic shear stress first increases linearly, then goes through a maximum, and starts to decrease as one increases the shear strain. This can be understood by inspecting Eq. (29) for the elastic shear stress. The applied magnetic field induces a nonzero magnetization, which, in turn, induces a nonzero elastic shear modulus. For small values of the shear strain $\Gamma$, in particular, for

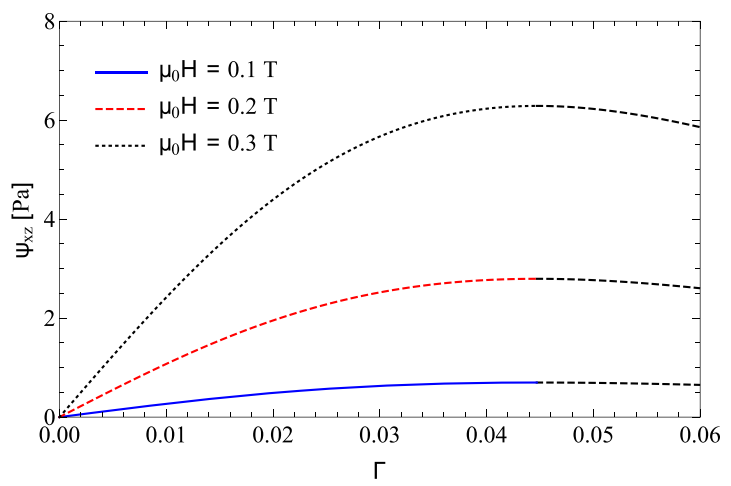

FIG. 2. The elastic shear stress as a function of the shear strain $\gamma_{2}=0$ at three different values of the applied magnetic field. The black dashed parts of the curves represent the unstable regime, where the elastic shear stress decreases with the shear strain.
$\Gamma \ll \alpha / \gamma_{2}$ and $\Gamma^{2} \ll \alpha / c_{2}$, the elastic shear stress [Eq. (29)] increases linearly with the shear strain

$$
\psi_{x z} \approx \frac{\mu_{0}^{2} H^{2}}{\alpha^{2}}\left(c_{2}-\frac{\gamma_{2}^{2}}{\alpha}\right) \Gamma
$$

and the initial slope of the elastic shear stress increases quadratically with the applied magnetic field.

As one increases the shear strain, the elastic shear stress saturates and finally decreases (Fig. 2). One reason for this is the magnetization, which decreases for increasing strains (see Fig. 3). Disregarding the magnetostrictive parameter $\gamma_{2}$ for the moment, the induced elastic shear stress reads (for any $\Gamma$ )

$$
\psi_{x z}=\mu_{0}^{2} H^{2} \frac{c_{2} \Gamma}{\left(\alpha+c_{2} \Gamma^{2}\right)^{2}} .
$$

Equation (33) provides a tool to determine the static coefficients $c_{2}$ and $\alpha$ from the comparison of the model to the measurements of the stress as a function of the strain. The stress-strain curve indeed has a maximum at

$$
\Gamma^{c}=\sqrt{\frac{\alpha}{3 c_{2}}},
$$

which is called the yield strain. Taking into account $\gamma_{2}$ perturbatively, e.g., for $\gamma_{2}^{2} \ll \alpha c_{2}$, it is shifted to higher strains (Fig. 4),

$$
\Gamma^{\text {yield }} \approx \Gamma^{c}\left(1+\frac{5}{8} \frac{\gamma_{2}^{2}}{\alpha c_{2}}\right)
$$

and does not depend on the magnetic field.

The value of the maximum elastic shear stress, the static yield stress, decreases with increasing $\gamma_{2}$ according to

$$
\psi_{x z}^{\text {yield }} \approx \frac{3 \sqrt{3}}{16} \sqrt{\alpha c_{2}}\left(1-\frac{3}{16} \frac{\gamma_{2}^{2}}{\alpha c_{2}}\right) \frac{\mu_{0}^{2} H^{2}}{\alpha^{2}} .
$$

In addition, the static yield stress scales quadratically with the applied magnetic field (as is also visible in Fig. 2), which is in agreement with experiments.

The magnetostriction is responsible for the tilting of the chains of magnetizable particles. The tilt angle $\theta$ can be calculated from Eqs. (30) and (31) and is proportional to $\gamma_{2}$,

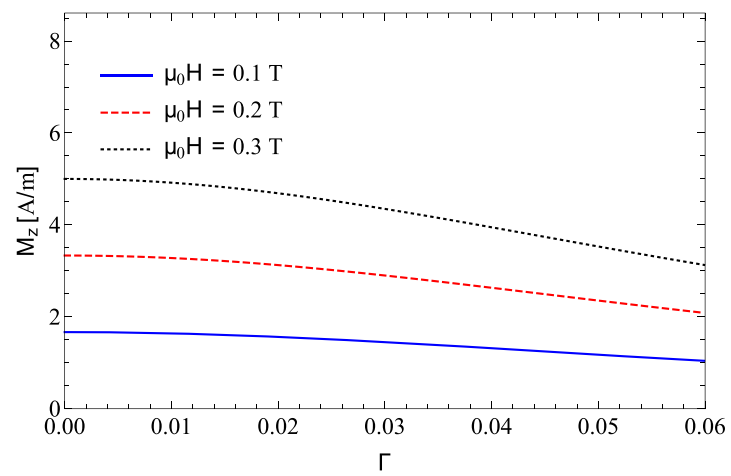

FIG. 3. The $z$ component of the magnetization $M_{z}$ as a function of the shear strain for $\gamma_{2}=0$ at three different values of the applied magnetic field. 


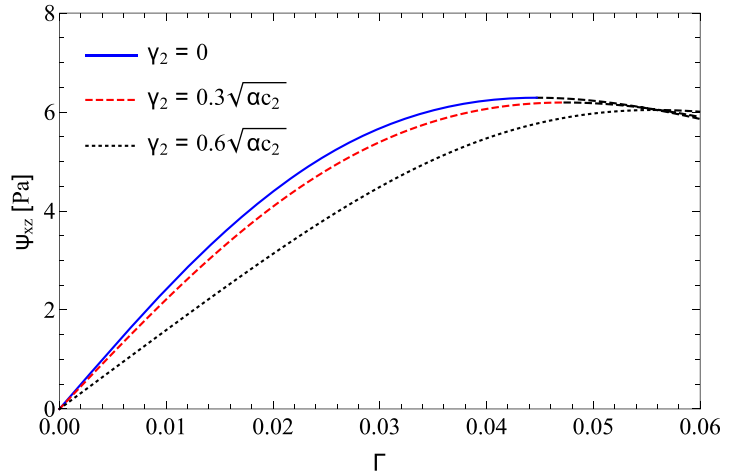

FIG. 4. The elastic shear stress vs shear strain for three different values of the magnetostriction coefficient $\gamma_{2}$ at a magnetic field $\mu_{0} H=0.3 \mathrm{~T}$. The black dashed parts of the curves represent the unstable regime, where the elastic shear stress decreases with the shear strain.

$$
\tan \theta=\frac{M_{x}}{M_{z}}=\frac{\gamma_{2} \Gamma}{\alpha+c_{2} \Gamma^{2}}
$$

with the maximum tilt value

$$
\tan \theta^{\max }=\frac{\gamma_{2}}{2 \sqrt{\alpha c_{2}}}
$$

which is taken at $\Gamma=\sqrt{\alpha / c_{2}}$.

We note, in passing, that given Eqs. (33) and (37), it is obvious that $\tan \theta$ and $\psi_{x z}$ do not have their maximum at the same $\Gamma$. As a consequence of the tilting of the chains, the elastic stress decreases as has been discussed, above. Experimentally, the maximum tilt angle of the chains with respect to the direction of the magnetic field is on the order of $10^{\circ}$, which implies $\gamma_{2} \approx 0.4 \sqrt{\alpha c_{2}} \approx 3.0 \times 10^{-1} \mathrm{~Pa} \mathrm{~A}^{-2} \mathrm{~m}^{2}$. This means that the approximation $\gamma_{2}^{2} \ll \alpha c_{2}$, which we used for some of the analytical results of this section, is quite appropriate.

\section{B. Effects of normal pressure}

A desirable property of MR fluids is a high static shear yield stress. This can to some extent be achieved by using a large magnetic field; however, due to the saturation of the magnetization, the static yield stress also saturates as one increases the magnetic field. In Refs. 51-54, it was found that, after the application of a magnetic field, compressing the MR fluid along the field direction strongly increases the static shear yield stress. Moreover, the static yield stress was found to be linearly dependent on the applied pressure $P$,

$$
\psi_{x z}^{\text {yield }}(P)=\psi_{x z}^{\text {yield }}(0)+k P,
$$

where $\psi_{x z}^{\text {yield }}(0)$ is the static yield stress without compression, and the slope $k$ was shown in Ref. 51 to be only very slightly increasing with the magnetic field.

Physically, the increase in the static shear yield stress can be explained by the fact that the compression pushes the chains of magnetizable particles to form thicker columns, which can better resist the shear forces.

We have found that the magnetostriction coefficient $\sim \gamma_{1}$ accounts for these experimental findings. Since the effect of $\gamma_{2}$ on the elastic shear stress has been discussed in Sec. III A, we will put $\gamma_{2}=0$ here. The external pressure $P$ corresponds to an external stress $\psi_{z z}=+P$, from which a compressive strain $\varepsilon_{z z}$ is induced via Eq. (8),

$$
\mathcal{E}_{z z}=+\frac{P}{\bar{c}_{1} \mathbf{M}^{2}},
$$

where the effective longitudinal elastic coefficient is $\bar{c}_{1}=c_{1}+2 c_{2}$. This strain comes in addition to the equilibrium compressive strain $\varepsilon_{z z}^{e q}$ due to the external field, discussed in Sec. II A.

As a result of the compression, the magnetization $M_{z}$ increases due to the magnetostrictive coupling $\sim \gamma_{1}\left(M_{x}\right.$ vanishes in the $\gamma_{2}=0$ approximation)

$$
M_{z} \approx \frac{\mu_{0} H}{\left(\alpha+c_{2} \Gamma^{2}\right)}+\frac{\gamma_{1}}{\bar{c}_{1} \mu_{0} H} P
$$

which we have linearized in the pressure.

An increase in the magnetization leads, according to Eq. (29), to an enhanced shear yield stress. In Fig. 5, we show the elastic shear stress as a function of the shear strain at three different values of the applied normal pressure.

Indeed, the elastic shear stress and its maximum (the static yield stress) increase linearly with the pressure (Fig. 6). For small values of the applied pressure, this is described by $k$ [Eq. (39)] which takes the form

$$
k \approx \frac{9}{32} \frac{\gamma_{1}}{\bar{c}_{1}} \sqrt{\frac{3 c_{2}}{\alpha}} .
$$

For typical experimental values of $k \approx 1 / 4$, one finds $\gamma_{1} \approx 1.2 \mathrm{~Pa} \mathrm{~A}^{-2} \mathrm{~m}^{2}$.

In addition, the static yield stress is shifted to higher strains. This shift of the critical strain is also linear in the pressure and can be observed in Fig. 5 for different values of the field.

For small values of the applied pressure, one gets

$$
\Gamma^{\text {yield }} \approx \Gamma^{c}+\frac{1}{2} \frac{\gamma_{1} \alpha}{\bar{c}_{1} \mu_{0}^{2} H^{2}} P
$$

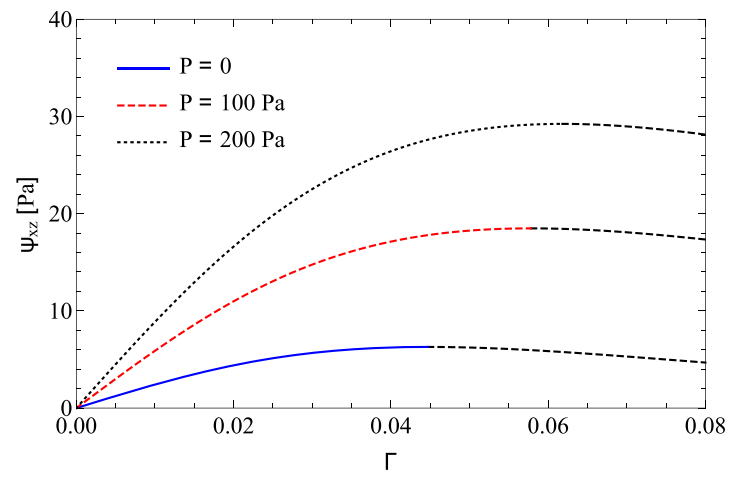

FIG. 5. Elastic shear stress as a function of shear strain using $\mu_{0} H=0.3 \mathrm{~T}$ at three different values of the applied compressive pressure. The black dashed parts of the curves represent the unstable regime, where the elastic shear stress decreases with the shear strain. 


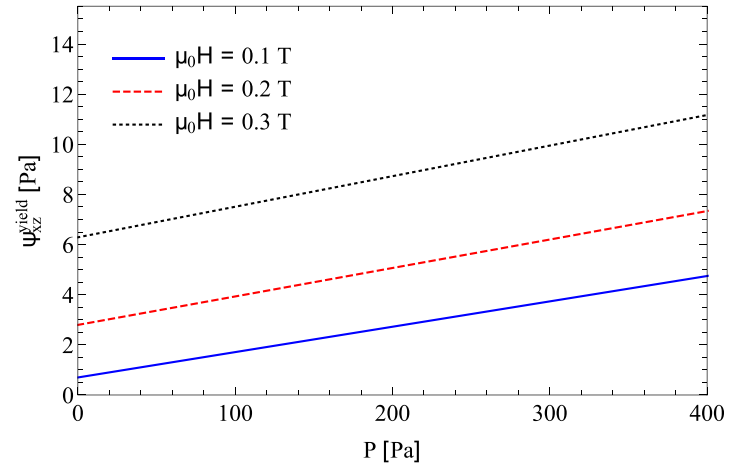

FIG. 6. Static yield stress as a function of the applied compressive pressure at three different values of the applied magnetic field.

showing also the $1 / H^{2}$ field dependence. By measuring the yield stress and the yield strain as a function of the pressure, one could, in principle, determine the coefficients $\gamma_{1}$ and $c_{1}$, while the coefficients $c_{2}$ and $\alpha$ could already be determined in the shear strain experiments (see Sec. III A).

\section{DYNAMIC DEFORMATIONS}

In this section, we investigate the effect of the magnetic field on the measured viscosities of MR fluids in the presence of an applied shear flow. Experimental results show a threshold behavior, where a finite stress, also called the dynamic shear stress, is needed to sustain a shear flow. In addition, MR fluids are typically found to be slightly shear thinning, which means the viscosity decreases as one increases the shear rate.

We assume simple shear with a linear velocity profile of the form $\mathbf{v}=\dot{\gamma} z \hat{\mathbf{e}}_{x}$, where the so-called shear rate is constant for a steady shear flow, $\dot{\gamma}=\dot{\gamma}_{0}$, Sec. IV A, and time-dependent for oscillatory flow, $\dot{\gamma}=\dot{\gamma}_{0} \cos (\omega t)$, Sec. IV B, with $\omega$ being the oscillatory frequency.

For shear flow, the dynamics of the magnetic degree of freedom, given in Sec. II, reads

$$
\begin{aligned}
-\frac{\partial}{\partial t} M_{x}= & b^{D} M_{x}\left(\alpha+\beta \mathbf{M}^{2}+4 c_{2} \varepsilon_{x z}^{2}\right)-\frac{1}{2}\left(1+2 c_{2}^{R}\right) M_{z} \dot{\gamma}, \\
-\frac{\partial}{\partial t} M_{z}= & b^{D} M_{z}\left(\alpha+\beta \mathbf{M}^{2}+4 c_{2} \varepsilon_{x z}^{2}\right)-b^{D} \mu_{0} H \\
& +\frac{1}{2}\left(1-2 c_{2}^{R}\right) M_{x} \dot{\gamma}=0
\end{aligned}
$$

where we have neglected $\gamma_{2}$, since it provided only corrections to the main results in the static shear strain case, and we expect the same for the presence of a shear flow. The couplings provided by the coefficient $d_{2}$ are comparable and are also neglected. We also take $b^{R}=0$, which ensures that the orientation of the magnetization (of the chains) does not deviate from the shear plane. Equations (44) and (45) are equivalent to those studied by Liu's group ${ }^{47,48}$ for magnetic liquids (where $\varepsilon_{x z}=0$ ), when we use the identification $2 c_{2}^{R}=\lambda_{2}$ and take $\beta=0$.

For the elastic degree of freedom, we get

$$
-\frac{\partial}{\partial t} \varepsilon_{x z}=\frac{4 c_{2}}{\tau_{2}} \varepsilon_{x z}-\frac{1}{2} \dot{\gamma}
$$

Finally, the total stress tensor, the momentum density current in Eq. (10) not only contains the elastic stress tensor $\psi_{i j}$ [Eq. (8)] but also the dissipative and reversible phenomenological parts $\sigma_{i j}^{D}$ [Eq. (17)] and $\sigma_{i j}^{R}$ [Eq. (23)]. It is given by

$$
\begin{aligned}
-\sigma_{x z}^{\mathrm{tot}}= & v_{2} \dot{\gamma}+2 c_{2} M^{2} \varepsilon_{x z}+\frac{1}{2}\left(1-2 c_{2}^{R}\right) \mu_{0} H M_{x} \\
& +2 c_{2}^{R} M_{x} M_{z}\left(\alpha+\beta \mathbf{M}^{2}+4 c_{2} \varepsilon_{x z}^{2}\right)
\end{aligned}
$$

and will be needed to set up the stress-strain rate relations, i.e., the apparent viscosity in Sec. IV A and the complex shear modulus in Sec. IV B.

\section{A. Steady shear flow}

In this section, we are only interested in stationary solutions of the dynamic equations. In that case, the left-hand sides of Eqs. (44)(46) are zero. This immediately allows us to relate the stationary shear strain to the applied shear flow

$$
\varepsilon_{x z}=\varepsilon_{z x}=\frac{\dot{\gamma} \tau_{2}}{8 c_{2}} .
$$

Of course, a stationary strain is only possible for a relaxing strain variable, while for permanent elasticity, the strain would increase indefinitely, when a constant flow is applied. Equation (48) represents the stationary balance between the increasing strain due to the shear flow and its relaxation due to the microscopic redistribution of the particles.

With this result, Eqs. (44) and (45) can be solved for the magnetization components $M_{x}$ and $M_{z}$ as functions of $\dot{\gamma}$ and $H$. This can only be done numerically due to the nonlinearities involved.

In Fig. 7, the shear stress Eq. (47) is plotted as a function of the shear rate for different values of the field. We also study the apparent viscosity, defined by

$$
\eta=\frac{-\sigma_{x z}^{\mathrm{tot}}}{\dot{\gamma}} .
$$

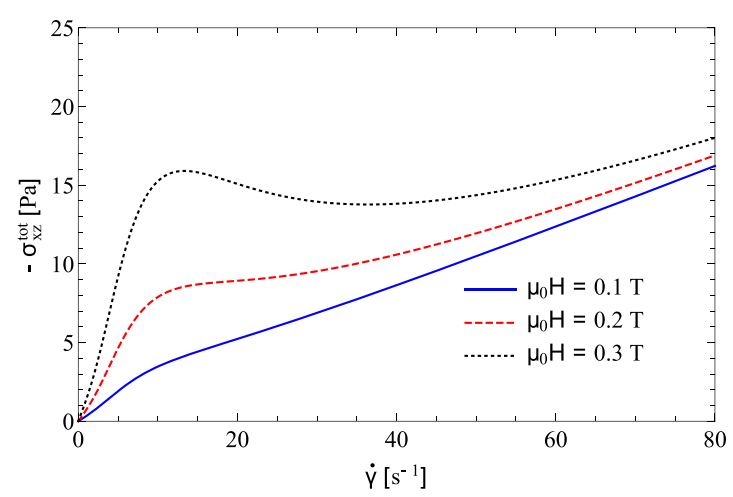

FIG. 7. The shear stress $-\sigma_{x z}^{\text {tot }}$ as a function of the steady shear rate at three different values of the applied magnetic field. 
There are basically three regimes. For very small shear rates, there is a steep linear increase in the stress and the slope (apparent viscosity $\eta$ ) strongly depends on the magnetic field. At very high shear rates, there is another (almost) linear stress increase, which is much smaller than in the initial regime and is, for very large shear rates, field independent. In between, the transition region shows a peak structure for higher magnetic fields. Reducing the external field, the peaklike structure diminishes until it simply denotes the transition between the low and high shear rate regimes. A peak structure, which gives rise to a local minimum at intermediate shear rates, can be explained by the induced elastic stress, described by the second term in Eq. (47). Initially, this stress increases linearly with the shear rate, which results in a steep initial slope of the flow curve [Eq. (50)]. At higher shear rates, the magnetization modulus starts to decrease inversely with the square of the shear rate, which decreases the elastic stress contribution toward zero. If the applied magnetic field is large enough, a peak structure will be observed.

The initial slope for small $\dot{\gamma}$ can be written approximately

$$
\eta_{\text {in }}=v_{2}+\left(\frac{1}{4} \tau_{2}+\frac{\left(1+2 c_{2}^{R}\right)^{2}}{4 b^{D}}\right) M_{0}^{2},
$$

where $M_{0}=\mu H_{0} / \alpha$ and $\nu_{2}$ is the viscosity (without field) due to the carrier fluid and the magnetizable particles suspended in it. The initial slope $\eta_{\text {in }}$ strongly increases with the external field, which is a prediction that could be tested in experiments. This is because shear flow reorients the columns of magnetizable particles due to the reversible coupling between flow and the magnetization via $c_{i j k}^{R}$ [Eq. (27)]. The $\mathrm{z}$-component of the magnetization is basically given by the magnetic field resulting in the field dependence of $\eta_{\text {in }}$. This effect is even bigger for smaller values of $b^{D}$, i.e., when the relaxation time of the magnetization is longer. This is the magnetic field dominated regime.

At a very high shear rate, the hydrodynamic regime is reached. Here, the influence of the magnetic field on the slope $\eta$ diminishes and finally, for $\dot{\gamma} \rightarrow \infty$, the flow curves converge to the same line, $-\sigma_{x z}^{\text {tot }}=v_{2} \dot{\gamma}$.

In the transition region, the stress obtains its (dynamic) yield stress value, $-\sigma_{x z}^{0}$, which is the stress needed to sustain flow.

There is some arbitrariness in the definition of the yield stress. Usually, it is read off from stress-strain relations as in Fig. 7 by extrapolating the high strain rate curves to zero strain rate. Or one could use as the yield stress the peak of the shear stress, or the shoulder, where the initial linear behavior turns into the final one. We determine this transition point as the shear rate at which the total shear stress, with the viscous contribution $\sim v_{2}$ subtracted, i.e., $-\sigma_{x z}^{\text {tot }}-v_{2} \dot{\gamma}$, attains a maximum. The dynamic yield stress is shown in Fig. 8 to be a quadratic function of the external field, $-\sigma_{x z}^{0}=$ $\Xi\left(\mu_{0} H\right)^{2}$ with $\Xi \approx 150 \mathrm{~A}^{2} \mathrm{~Pa}^{-1} \mathrm{~m}^{-2}$.

We have used the same set of parameters as in Sec. III, discussing the static deformations. It should be noted that a higher value of the yield stress may be obtained by using, for example, lower values of $\alpha$, which as a consequence increases the magnetic susceptibility. This stress also increases with increasing $b^{D}$, while the dependence of the yield stress on $\tau_{2}$ increases for low values and then starts to decrease for larger values of $\tau_{2}$.

Beyond the initial steep rise, the shear stress is often described approximately by a Bingham model

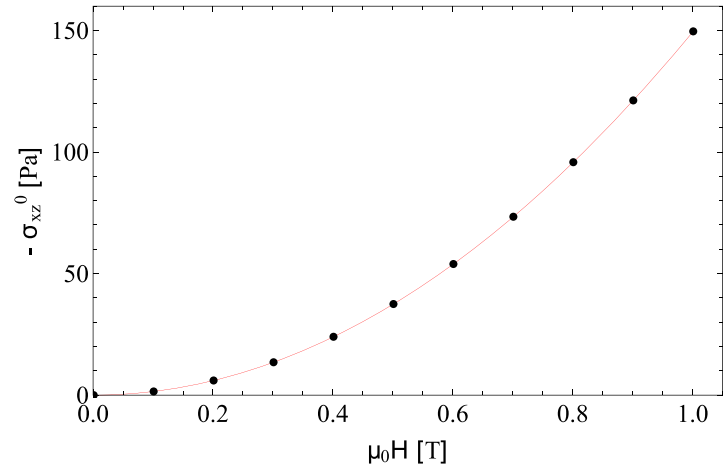

FIG. 8. The yield shear stress $-\sigma_{x z}^{0}$ as a function of the magnetic field.

$$
-\sigma_{x z}=-\sigma_{x z}^{0}+\eta_{\infty} \dot{\gamma}
$$

where a Newtonian viscous contribution is combined with the yield stress $-\sigma_{x z}^{0}$. Real MR fluids generally deviate from the Bingham model, in particular, for higher magnetic fields. Somewhat better fits to the experimental data can be achieved by using the so called Casson or Herschel-Bulkley models, ${ }^{55}$ which are frequently more suitable for flow curves that are not linear. In Fig. 9, it is shown, for the highest field case, that the apparent viscosity in our description is lower than in the Bingham model, demonstrating shear thinning. This effect is smaller for intermediate fields and almost invisible for low fields. This is in accordance with experimental findings, where the Mason number ${ }^{40,56}$ is often found to be slightly lower than 1 , indicating shear thinning. ${ }^{57-63}$ We mention that a model that takes into account the anisotropy together with the additional dynamic interplay of the relative rotations between the magnetization and the elastic network may produce a stress-strain curve that is closer to the Bingham model, Eq. (51). Another possibility is to consider the 2-fluid description of the magnetizable particulate phase and the solvent, but this is beyond the scope of this work.

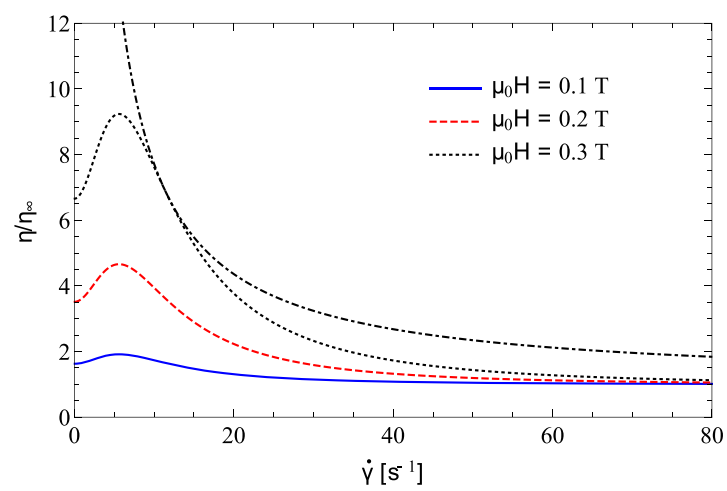

FIG. 9. The apparent viscosity $\eta$, scaled by $\eta_{\infty}$, as a function of the shear rate for three different values of the applied magnetic field. Bingham behavior is shown as a dashed-dotted line for the high field case. The shear thinning of the apparent viscosity at intermediate shear rates compared to the Bingham model is obvious. For $\dot{\gamma} \rightarrow \infty$, all curves converge at $\eta / \eta_{\infty} \rightarrow 1$. 


\section{B. Oscillatory shear flow}

In this section, we study the viscoelastic properties of MR fluids in the linear response regime. We impose an oscillatory deformation along the $x$ axis, $\mathbf{v}(z)=\dot{\gamma} e^{-\mathrm{i} \omega t} z \hat{\mathbf{e}}_{x}+$ c.c., with $\omega$ being the frequency of the oscillation and where c.c. denotes the complex conjugate. A measure of the viscoelastic properties is the complex shear modulus $G=G^{\prime}+\mathrm{i} G^{\prime \prime}$, defined as the ratio of the shear stress $\sigma_{x z}^{\text {tot }}$ [Eq. (47)] and the imposed strain $-\gamma \equiv \dot{\gamma} / \mathrm{i} \omega, G=\sigma_{x z}^{\text {tot }} / \gamma$. The real, $G^{\prime}$, and the imaginary part, $G^{\prime \prime}$, are the storage and the loss modulus, describing the reactive and dissipative response, respectively. We note that $\gamma$ is not identical to the $\Gamma$ of Sec. III, as can be seen from Eq. (46).

To calculate the complex shear modulus, we linearize Eqs. (44)(46) around equilibrium, where the strain field $\varepsilon_{i j}$ is zero and the magnetization points along the $z$ axis, $\mathbf{M}=M_{0} \hat{\mathbf{e}}_{z}$, with $M_{0}=\mu_{0} H / \alpha$ for low magnetic fields. For the relevant variables $M_{x}, M_{z}$, and $\varepsilon_{x z}$, we use the ansatz $M_{x}=M_{x}^{(0)} e^{-\mathrm{i} \omega t}+$ c.c., $M_{z}=M_{0}+\left(M_{z}^{(0)} e^{-\mathrm{i} \omega t}+\right.$ c.c. $)$, and $\varepsilon_{x z}=\varepsilon_{x z}^{(0)} e^{-\mathrm{i} \omega t}+$ c.c., with $M_{x}^{(0)}, M_{z}^{(0)}$, and $\varepsilon_{x z}^{(0)}$ being the corresponding amplitudes, which are, in general, complex quantities. The frequency is assumed to be sufficiently small so that the linear velocity profile is established at any time.

The characteristic time scales are $\tau_{\mathrm{el}}=\tau_{2} /\left(4 c_{2}\right)$, corresponding to the relaxing strain, and $\tau_{m}=1 /\left(b^{D} \alpha\right)$, corresponding to the magnetization relaxation. For the parameter values used in this section, the time scales are almost equal, $\tau_{\mathrm{el}} \lesssim \tau_{m}$.

Experimental results ${ }^{63-65}$ show that the storage modulus is considerably larger than the loss modulus at intermediate frequencies and that both increase with increasing magnetic field, which we could qualitatively reproduce (see Fig. 10). This means that the system behaves more like a solid than a liquid, which is expected, since a small amplitude shear oscillation can only slightly influence the strength of the columns. For smaller frequencies, the numerical results show that the system behaves, as expected, more like a liquid than a solid (Fig. 10).

For low frequencies, the storage modulus increases quickly with frequency and then saturates for larger frequencies. ${ }^{64,65}$ This can be seen in Fig. 10. The initial rise of the storage modulus is quadratic in the frequency

$$
G^{\prime} \approx\left(\frac{\alpha}{4}\left(1+2 c_{2}^{R}\right)^{2} \tau_{m}^{2}+c_{2} \tau_{\mathrm{el}}^{2}\right) M_{0}^{2} \omega^{2},
$$

while for larger frequencies, the plateau value

$$
G_{\infty}^{\prime}=\left(\frac{\alpha}{4}\left(1+2 c_{2}^{R}\right)^{2}+c_{2}\right) M_{0}^{2}
$$

is reached. The increase in the storage modulus at low frequencies is not reported often, which is either because many of the experiments have not gone quite to the low frequency regime, or the characteristic frequency where one observes such an effect, is not experimentally accessible for many setups. In Ref. 64, a saturation of the storage modulus is observed at a frequency of about $1 \mathrm{~Hz}$.

The loss modulus, on the other hand, has a slightly more complicated behavior. Theoretical results show that the loss modulus increases linearly for low frequencies

$$
G^{\prime \prime} \approx \eta_{\text {in }} \omega,
$$

where $\eta_{\text {in }}$ is the initial slope of the steady shear stress [Eq. (50)]. After a maximum, it starts to decrease, and at intermediate frequencies, it
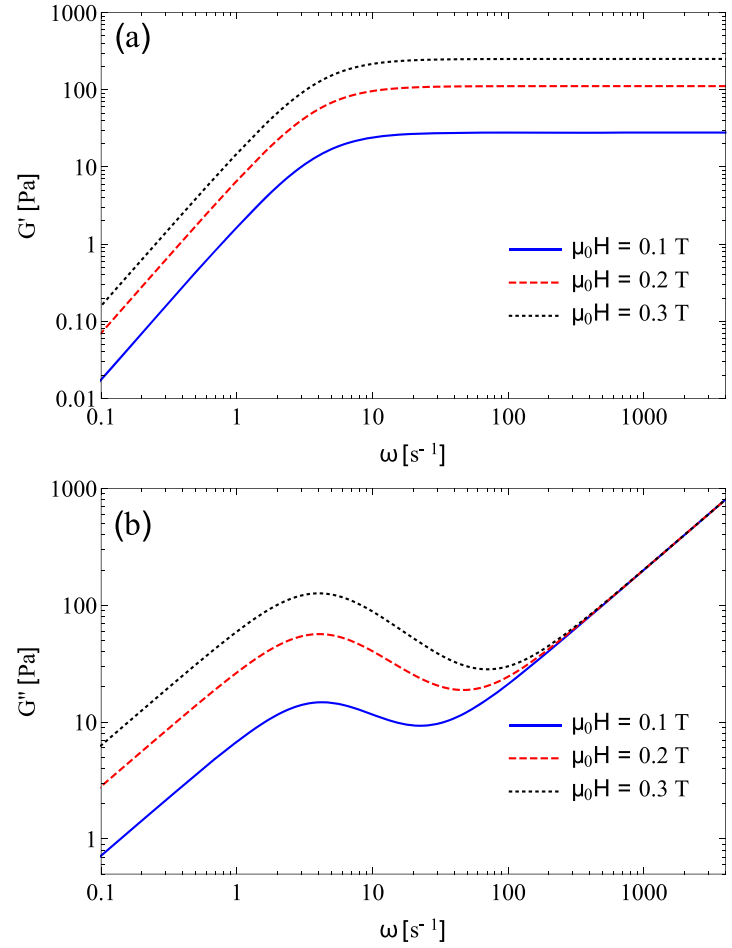

FIG. 10. (a) Storage modulus $G^{\prime}$ and (b) loss modulus $G^{\prime \prime}$ as a function of the frequency at three different values of the applied magnetic field.

passes a minimum before it increases, finally. The location of the maximum is at

$$
\omega_{\max } \approx 1 / \tau_{\mathrm{el}}
$$

and is independent of the magnetic field.

The minimum of the loss modulus at intermediate frequencies shifts to larger frequencies as one increases the magnetic field [Fig. 10(b)], which is detected in certain experiments. ${ }^{6,64}$ For $\omega^{2} \tau_{m}^{2} \gg 1$ and $\omega^{2} \tau_{\mathrm{el}}^{2} \gg 1$, the minimum is at

$$
\omega_{\min } \approx \frac{M_{0}}{\sqrt{v_{2}}} \sqrt{\frac{4 c_{2}^{2}}{\tau_{2}}+\frac{1}{4}\left(1+2 c_{2}^{R}\right)^{2} b^{D} \alpha^{2}},
$$

where the frequency of the minimum $\omega_{\min }$ shifts linearly with the field.

The final, asymptotic behavior of the loss modulus for $\omega \rightarrow \infty$ is described by the viscosity $v_{2}$,

$$
G^{\prime \prime} \asymp v_{2} \omega .
$$

The maximum of the loss modulus at small frequencies has not been reported often, perhaps due to the experimental limitations at lower frequencies. There are certain indications that such a maximum exists.

We found that a simple relation exists for the master curves $g^{\prime}$ and $g^{\prime \prime}$ of $G^{\prime}$ and $G^{\prime \prime}$, respectively. The storage modulus has to be rescaled by a factor of $\mu_{0} H^{2}, g^{\prime}(\omega)=G^{\prime}(\omega) / \mu_{0} H^{2}$, while the viscosity term needs to be subtracted first in the loss modulus, $g^{\prime \prime}(\omega)=\left(G^{\prime \prime}(\omega)\right.$ $\left.-v_{2} \omega\right) / \mu_{0} H^{2}$. The storage and the loss moduli can be at any given 


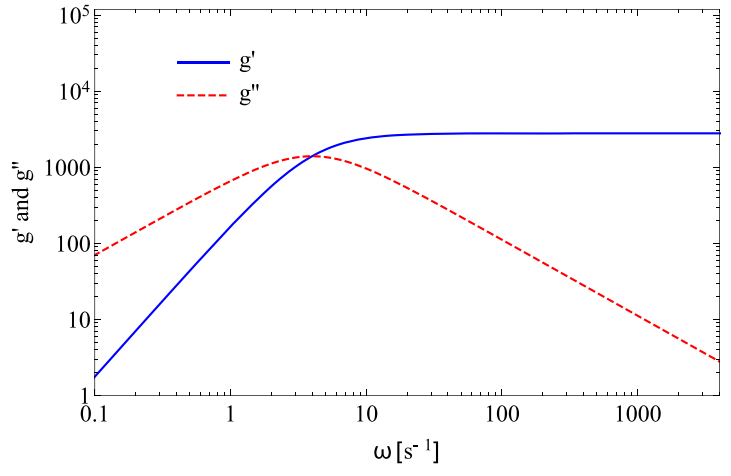

FIG. 11. The master curves $g^{\prime}$ and $g^{\prime \prime}$ as a function of the frequency $\omega$ corresponding to the storage $G^{\prime}$ and the loss modulus $G^{\prime \prime}$, respectively.

magnetic field $H$ reduced exactly to the curves $g^{\prime}(\omega)$ and $g^{\prime \prime}(\omega)$, which is not surprising because all contributions in the stress tensor [Eq. (47)] apart from the viscosity term $\sim v_{2}$, are proportional to the square of the magnetic field. Since the characteristic time scales $\tau_{m}$ and $\tau_{\mathrm{el}}$ are independent of the magnetic field, the master curves are obtained without the need to rescale the frequency variable (Fig. 11).

We now discuss the dependence of the absolute value of the complex shear modulus $|G|=\sqrt{\left(G^{\prime}\right)^{2}+\left(G^{\prime \prime}\right)^{2}}$ on the frequency. After the initial linear increase, governed by the loss modulus [Eq. (54)], there is a plateau at intermediate frequencies, which is basically given by the plateau of the storage modulus $G_{\infty}^{\prime}$ [Eq. (53)], since the loss modulus $G^{\prime \prime}$ is much smaller there. For high frequencies, the loss modulus is dominating again and $|G|$ increases according to Eq. (57). This final increase is best visible in Fig. 12 for low fields.

This scenario applies to the case of (almost) equal elastic and magnetic time scales. If these time scales are sufficiently well separated, a somewhat different behavior of $|G|$ is found (Fig. 13). After the very steep initial rise, a very narrow plateau is found at rather low frequencies, which is approximately of height $c_{2} M_{0}^{2}$ (for $\tau_{\mathrm{el}} \ll \tau_{m}$ ) and $\frac{1}{4}\left(1+2 c_{2}^{R}\right)^{2} \alpha M_{0}^{2}$ (for $\tau_{\mathrm{el}} \gg \tau_{m}$ ). At intermediate frequencies, $|G|$

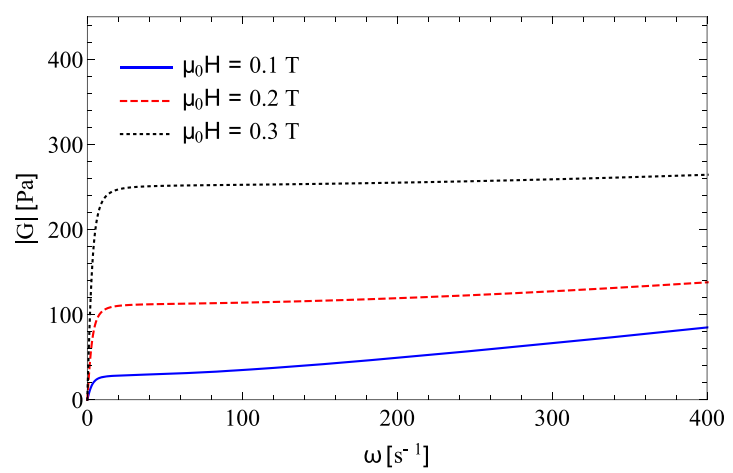

FIG. 12. $|G|$ as a function of the frequency at three different values of the applied magnetic field.

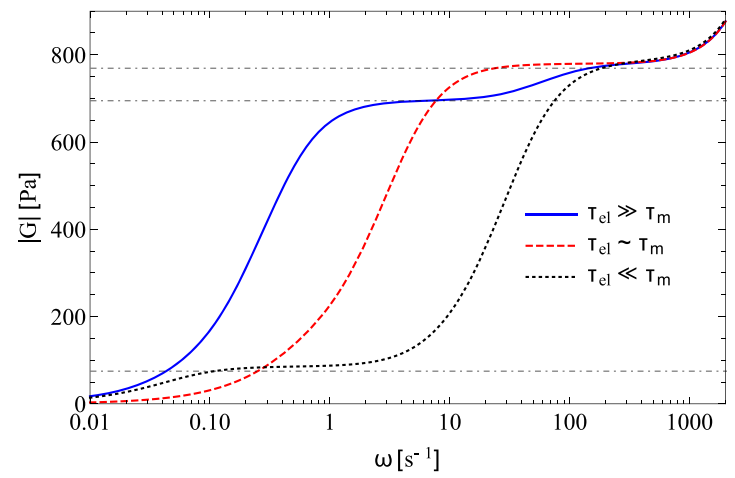

FIG. 13. Shear modulus $|G|$ as a function of the frequency in a semilog plot at $\mu_{0} H=0.5 \mathrm{~T}$ for three different (arbitrary) choices of the time scales $\tau_{\mathrm{el}}$ and $\tau_{m}$. The light black dashed horizontal lines denote the values of the plateaus for the different cases. To enhance the visibility of the lowest plateau, $c_{2}^{R}=4$ has been chosen in this plot.

gently increases to the combined plateau $G_{\infty}^{\prime}$ [Eq. (53)] and finally converges to the asymptotic behavior independent of the relaxation times.

In polymer dynamics, the empirical Cox-Merz rule is often very well fulfilled. It allows us to estimate shear stresses when a steady shear is imposed from data obtained by small amplitude oscillatory strain rate experiments. This rule states that at a given frequency $\omega$, the modulus $|G|$ is identical to the shear stress, $-\sigma_{x z}^{\text {tot }}$, under a steady shear rate $\dot{\gamma}=\omega$. It is trivially fulfilled for $\dot{\gamma} \rightarrow \infty$ and $\omega \rightarrow \infty$, where $|G| \asymp v_{2} \omega$ and $-\sigma_{x z}^{\text {tot }} \asymp v_{2} \dot{\gamma}$. Similarly, for very small $\omega,|G| \approx G^{\prime \prime} \sim \omega$ [Eq. (54)] increases the same way as $-\sigma_{x z}^{\text {tot }} \sim \dot{\gamma}$. Applying the Cox-Merz rule to the yield shear stress, however, would lead to

$$
-\sigma_{x z}^{0}=\left(\frac{\left(1+2 c_{2}^{R}\right)^{2}}{4}+\frac{c_{2}}{\alpha}\right) \alpha M_{0}^{2} \approx 2800\left(\mu_{0} H\right)^{2}
$$

for the stationary yield stress, which is, though, much larger than the value of $150\left(\mu_{0} H\right)^{2}$ found in Sec. IV A (Fig. 9). This shows that the Cox-Merz rule is not obeyed in MR fluids. This difference is probably due to the columnar structures, which are not destroyed in the small amplitude oscillatory shear, but are destroyed in steady shear. Equations (56) and (58) can be used to determine the dynamic coefficients $c_{2}^{R}, b^{D}$, and $\tau_{2}$ or serve as an additional method to determine the static parameter $c_{2}$.

\section{SUMMARY AND PERSPECTIVE}

In this study, a simple macroscopic model for the MR fluids has been proposed. We tested the model on several simple experimental configurations, such as the influence of a magnetic field or a normal pressure on the static shear deformations as well as simple shear flow in the steady and the oscillatory regime.

In order to model the effects of the chains of magnetizable particles, which are observed in experiments, and to capture the solidlike properties, we included as the macroscopic variables the magnetization and the strain field. We have shown that the inclusion of these variables well explains certain experimental facts, such 
as the existence of a static yield stress or the influence of a normal pressure on the static yield stress. We have successfully reproduced the quadratic field dependence of the static yield stress with the appropriate critical strain being independent of the magnetic field. This was made possible by a (quadratic) dependence of the elastic moduli on the magnetization, which is motivated by the fact that the solidlike properties of MR fluids are due to the magnetic field.

Furthermore, we probed the dynamics of our model by investigating the stresses that arise by applying a shear flow. Here, our assumption that the strain relaxation coefficients are proportional to the magnetization squared comes into play, which is motivated by the fact that the elastic network relaxes on longer time scales in larger magnetic fields. First, we applied a stationary shear flow, leading to the flow curves describing the shear stress as a function of the shear rate. For intermediate values of the shear rate, a maximum, the dynamic yield stress, was obtained and the flow curve could almost be described by the Bingham law. However, deviations were found indicating shear thinning, in particular, for higher magnetic fields.

We also studied the effects of an imposed oscillatory shear flow. We showed that the complex shear modulus as a function of the frequency exhibits a plateau. The values of the plateau are related to the elastic shear modulus or to the hydrodynamic coupling between magnetization and flow, or to both, depending on whether the elastic relaxation time is much larger, or much smaller, or almost equal to the magnetic relaxation time, respectively. Applying the Cox-Merz rule, which compares the plateau regime with the dynamic yield stress of the stationary case, revealed that the Cox-Merz rule is not fulfilled by our model.

We derive equations and present expressions that can be used for measuring certain phenomenological coefficients. Examples are the elastic and magnetostriction coefficients, which could be determined from measurements of the stress-strain curves and the maximum tilt angles of the chains. We also discuss the possibilities to determine the dynamic coefficients by measuring, for example, the initial slope of the stress as a function of the shear rate, the shift of the minimum in the loss modulus $G^{\prime \prime}$ as a function of the magnetic field, or by measuring the plateau values of the shear modulus $|G|$. We have also shown that a simple master curve may be generated for the storage and the loss modulus.

In the next step, it is desirable to capture various aspects of pattern formation in MR fluids. Examples of these patterns include the formation of the columns themselves, the thickening of the columns under a pressure force, ${ }^{66}$ the stripe formation under shear flow, ${ }^{67-69}$ or structures formed in a rotating magnetic field. ${ }^{70}$ A macroscopic 2fluid model has been presented in Ref. 71, but has never been applied to magnetic systems such as MR fluids. In this case, one would model the MR fluid with the solvent phase and the particle phase as two separate fluid phases.

As a perspective, we mention the generalizations to large magnetic fields for which the approximation used in the present no longer applies. In addition, the role of relative rotations between the magnetization, $M_{i}$, and the elastic matrix should also be investigated. Relative rotations could also contribute to the tilting of the chains, complementing the magnetostrictive effects discussed in Sec. III A.

\section{ACKNOWLEDGMENTS}

We thank one of the referees for asking about the existence of the master curve in the dynamic oscillatory shear flow. Partial support of this work through the Schwerpunktprogramm SPP 1681 "Feldgesteuerte Partikel-Matrix-Wechselwirkungen: Erzeugung, skalenübergreifende Modellierung und Anwendung magnetischer Hybridmaterialien" of the Deutsche Forschungsgemeinschaft is gratefully acknowledged, as well as the support of the Slovenian Research Agency, Grant No. P1-0055 (D.S.).

\section{REFERENCES}

${ }^{1}$ J. Rabinow, Trans. Am. Inst. Electr. Eng. 67, 1308 (1948).

${ }^{2}$ B. J. Park, F. F. Fang, and H. J. Choi, Soft Matter 6, 5246 (2010).

${ }^{3}$ J. de Vicente, D. Klingenberg, and R. Hidalgo-Alvarez, Soft Matter 7, 3701 (2011).

${ }^{4}$ A. Zubarev, L. Iskakova, M. T. Lopez-Lopez, P. Kuzhir, and G. Bossis, J. Rheol. 58, 1673 (2014).

${ }^{5}$ J. A. Ruiz-Lopez, J. C. Fernandez-Toledano, D. J. Klingenberg, R. HidalgoAlvarez, and J. de Vicente, J. Rheol. 60, 61 (2016).

${ }^{6}$ G. Bossis, O. Volkova, Y. Grasselli, and A. Ciffreo, Front. Mater. 6, 4 (2019).

${ }^{7}$ J. M. Ginder and L. C. Davis, Appl. Phys. Lett. 65, 3410 (1994).

${ }^{8}$ J. M. Ginder, in Encyclopedia of Applied Physics, edited by G. L. Trigg (VCH, Weinheim, 1996), Vol. 16, p. 487.

${ }^{9}$ G. Bossis, E. Lemaire, O. Volkova, and H. Clercx, J. Rheol, 41, 687 (1997).

${ }^{10}$ G. Bossis, O. Volkova, S. Lacis, and A. Meunier, Lect. Notes Phys. 594, 202 (2002).

${ }^{11}$ D. J. Klingenberg and C. F. Zukoski, Langmuir 6, 15 (1990).

${ }^{12}$ G. L. Gulley and R. Tao, Phys. Rev. E 48, 2744 (1993).

${ }^{13}$ T. C. Halsey, J. E. Martin, and D. Adolf, Phys. Rev. Lett. 68, 1519 (1992).

${ }^{14}$ S. Melle and J. E. Martin, J. Chem. Phys. 118, 9875 (2003).

${ }^{15}$ J. E. Martin, J. Odinek, T. C. Halsey, and R. Kamien, Phys. Rev. E 57, 756 (1998).

${ }^{16}$ R. T. Bonnecaze and J. F. Brady, J. Chem. Phys. 96, 2183 (1992).

${ }^{17}$ P. O. Brunn and B. Abu-Jdayil, Z. Angew. Math. Mech. 78, 97 (1998).

${ }^{18}$ Y. M. Shkel and D. J. Klingenberg, J. Rheol. 43, 1307 (1999).

${ }^{19}$ A. T. Horvath, D. J. Klingenberg, and Y. M. Shkel, Int. J. Mod. Phys. B 16, 2690 (2002).

${ }^{20}$ R. E. Rosensweig, J. Rheol. 39, 179 (1995).

${ }^{21}$ J. W. Zhang, X. Q. Gong, C. Liu, W. Wen, and P. Sheng, Phys. Rev. Lett. 101, 194503 (2008).

${ }^{22}$ P. Sheng and W. Wen, Solid State Commun. 150, 1023 (2010).

${ }^{23}$ K. von Pfeil, M. D. Graham, D. J. Klingenberg, and J. F. Morris, Phys. Rev. Lett. 88, 188301 (2002).

${ }^{24}$ K. von Pfeil, M. D. Graham, D. J. Klingenberg, and J. F. Morris, J. Appl. Phys. 93, 5769 (2003).

${ }^{25} \mathrm{~K}$. von Pfeil and D. J. Klingenberg, J. Appl. Phys. 96, 5341 (2004).

${ }^{26} \mathrm{H}$. Pleiner and H. R. Brand, "Hydrodynamics and electrohydrodynamics of nematic liquid crystals," in Pattern Formation in Liquid Crystals, edited by A. Buka and L. Kramer (Springer, New York, 1996).

${ }^{27}$ W. E. Isler and D. Y. Chung, J. Appl. Phys. 49, 1812 (1978).

${ }^{28}$ H. Pleiner and H. R. Brand, J. Magn. Magn. Mater. 85, 125 (1990).

${ }^{29}$ H. R. Brand and H. Pleiner, Phys. Rev. Lett. 86, 1385 (2001).

${ }^{30}$ A. Menzel, H. Pleiner, and H. R. Brand, J. Appl. Phys. 105, 013503 (2009).

${ }^{31}$ A. Menzel, H. Pleiner, and H. R. Brand, Eur. Phys. J. E 30, 371 (2009).

${ }^{32}$ E. Jarkova, H. Pleiner, H.-W. Müller, and H. R. Brand, Phys. Rev. E 68, 041706 (2003).

${ }^{33}$ S. Bohlius, H. R. Brand, and H. Pleiner, Phys. Rev. E 70, 061411 (2004).

${ }^{34}$ H. Temmen, H. Pleiner, M. Liu, and H. R. Brand, Phys. Rev. Lett. 84, 3228 (2000).

${ }^{35}$ H. Pleiner, M. Liu, and H. R. Brand, Rheol. Acta 39, 560 (2000).

${ }^{36}$ H. R. Brand, H. Pleiner, and D. Svenšek, Rheol. Acta 57, 773 (2018). 
${ }^{37}$ S. R. de Groot and P. Mazur, Nonequilibrium Thermodynamics (North Holland, Amsterdam, 1962).

${ }^{38}$ W. H. Li, H. Du, G. Chen, and S. H. Yeo, Mater. Sci. Eng. A 333, 368 (2002).

${ }^{39}$ J. Claracq, J. Sarrazin, and J.-P. Montfort, Rheol. Acta 43, 38 (2004).

${ }^{40}$ Y. Otsubo and K. Edamura, J. Rheol. 38, 1721 (1994).

${ }^{41}$ Z. Wang, K. Shahrivar, and J. de Vicente, J. Rheol. 58, 1725 (2014).

${ }^{42}$ K. D. Weiss, J. D. Carlson, and D. A. Nixon, J. Intell. Mater. Syst. Struct. 5, 772 (1994).

${ }^{43}$ B. D. Chin, J. H. Park, M. H. Kwon, and O. O. Park, Rheol. Acta 40, 211 (2001).

${ }^{44}$ Y. Yang, L. Li, and G. Chen, Rheol. Acta 48, 457 (2009).

${ }^{45}$ S. S. Deshmukh and G. H. McKinley, in Proceedings of XIVth International Congress on Rheology, Seoul, South Korea, August 2004.

${ }^{46}$ H. W. Müller and M. Liu, Phys. Rev. E 64, 061405 (2001).

${ }^{47}$ O. Müller, D. Hahn, and M. Liu, J. Phys.: Condens. Matter 18, S2623 (2006).

${ }^{48}$ S. Mahle, P. Ilg, and M. Liu, Phys. Rev. E 77, 016305 (2008).

${ }^{49}$ S. Odenbach and H. W. Müller, Phys. Rev. Lett. 89, 037202 (2002).

${ }^{50}$ S. Odenbach and H. W. Müller, J. Magn. Magn. Mater. 289, 242 (2005).

${ }^{51}$ X. Tang, X. Zhang, R. Tao, and Y. M. Rong, J. Appl. Phys. 87, 2634 (2000).

${ }^{52}$ X. Z. Zhang, X. L. Gong, P. Q. Zhang, and Q. M. Wang, J. Appl. Phys. 96, 2359 (2004).

${ }^{53}$ S. A. Mazlan, N. B. Ekreem, and A. G. Olabi, J. Mater. Process. Technol. 201, 780 (2007).

${ }^{54}$ H. Wang, D. Cheng, J. Kan, C. Gao, and W. Ziao, J. Intell. Mater. Syst. Struct. 22, 811 (2011).
${ }^{55}$ D. Susan-Resiga, J. Intell. Mater. Syst. Struct. 20, 1001 (2007).

${ }^{56}$ D. J. Klingenberg, J. C. Ulicny, and M. A. Golden, J. Rheol. 51, 883 (2007).

${ }^{57}$ D. W. Felt, M. Hagenbuchle, J. Liu, and J. Richard, J. Intell. Mater. Syst. Struct. 7, 589 (1996).

${ }^{58}$ B. J. de Gans, H. Hoekstra, and J. Mellema, Faraday Discuss. 112, 209 (1999).

${ }^{59}$ O. Volkova, G. Bossis, M. Guyot, V. Bashtovoi, and A. Reks, J. Rheol. 44, 91 (2000).

${ }^{60}$ Y. D. Liu, F. F. Fang, and H. J. Choi, Colloid Polym. Sci. 289, 1295 (2011).

${ }^{61}$ Y. Rabbani, M. Ashtiani, and S. H. Hashemabadi, Soft Matter 11, 4453 (2015).

${ }^{62}$ Y. Fu, J. Yao, H. Zhao, G. Zhao, Z. Wan, and Y. Qiu, Smart Mater. Struct. 27, 125001 (2018).

${ }^{63}$ J. H. Lee, Q. Lu, J. Y. Lee, and H. J. Choi, Polymers 11, 219 (2019).

${ }^{64}$ G. Wang, Y. Ma, G. H. Cui, N. N. Li, and X. F. Dong, Soft Matter 14, 1917 (2018).

${ }^{65}$ I. Arief and P. K. Mukhopadhyay, J. Alloys Compd. 696, 1053 (2017).

${ }^{66}$ R. Tao, J. Phys.: Condens. Matter 13, R979 (2001).

${ }^{67}$ S. Cutillas and G. Bossis, Eur. Phys. Lett. 40, 465 (1997).

${ }^{68}$ S. Cutillas, G. Bossis, and A. Cebers, Phys. Rev. E 57, 804 (1998).

${ }^{69}$ O. Volkova, S. Cutillas, P. Carletto, G. Bossis, A. Cebers, and A. Meunier, J. Magn. Magn. Mater. 201, 66 (1999).

${ }^{70}$ P. Carletto, G. Bossis, and A. Cebers, Int. J. Mod. Phys. B 16, 2279 (2002).

${ }^{71}$ H. Pleiner and J. L. Harden, AIP Conf. Proc. 708, 46 (2004); e-print arXiv:condmat/0404134. 